\title{
ANÁLISE DA VARIAÇÃO ANATÔMICA DO MÚSCULO PALMAR LONGO EM SERES HUMANOS
}

\section{ARTIGO DE REVISÃO}

SPAZIANI, Amanda Oliva ${ }^{1}$

ANDREANI, Giovanna ${ }^{2}$

ARAUJO, Isabella Moreira ${ }^{3}$

MÜLLER, Karla Maria ${ }^{4}$

SPAZIANI, Luis Carlos 5

RAMOS, Rogério Rodrigo ${ }^{6}$

${ }^{1}$ Graduanda em Medicina pela Universidade Brasil - Fernandópolis, Brasil.

${ }^{2}$ Graduanda em Medicina pela Universidade Brasil - Fernandópolis, Brasil.

${ }^{3}$ Graduanda em Medicina pela Universidade Brasil - Fernandópolis, Brasil.

${ }^{4}$ Graduanda em Medicina pela Universidade Brasil - Fernandópolis, Brasil.

${ }^{5}$ Engenheiro Graduado em Engenharia de Materiais pela Universidade Federal de São Carlos (UFSCar) - São Carlos, Brasil. Especialista em Docência Universitária pela FACSENAC - Brasil. Mestre em Economia do Meio Ambiente pela Universidade de Brasília (UnB) - Brasília, Brasil.

${ }^{6}$ Enfermeiro Graduado em Enfermagem e Especialista em Urgência e Emergência pela Fundação Educacional de Fernandópolis - Fernandópolis, Brasil. Mestre em Engenharia Biomédica pela Universidade Camilo Castelo Branco - Fernandópolis, Brasil. Doutor em Ciências da Saúde pela Faculdade de Medicina de São José do Rio Preto (FAMERP). Professor do Curso de Medicina da Universidade Brasil Fernandópolis, Brasil. 
TALIARI, Jean Donizete Silveira ${ }^{7}$

SPAZIANI, Amanda Oliva. Et al. Análise da variação anatômica do músculo palmar longo em seres humanos. Revista Científica Multidisciplinar Núcleo do Conhecimento. Ano 04, Ed. 07, Vol. 14, pp. 24-38. Julho de 2019. ISSN: 2448-0959

\section{RESUMO}

Objetivo: Foi analisar a variação anatômica do Músculo Palmar Longo em seres humanos encontrados em três estudos nacionais. Metodologia: Foi realizada uma revisão de literatura. Foram analisados dados de publicações que usaram método de Schaeffer para avaliar a presença do músculo. Em um dos estudos, foram analisados 240 discentes da Universidade Federal dos Vales do Jequitinhonha e Mucuri, já em Maringá, participaram 200 voluntários. Enquanto, em Jataí, a pesquisa foi composta por 740 participantes. Conclusão: Foi possível concluir que o músculo palmar longo está presente na maioria da população e com uma incidência maior no sexo masculino.

Palavras-chave: Variação anatômica, músculo palmar longo, método de Schaeffer.

\section{INTRODUÇÃO}

Pode ser considerada uma variação anatômica qualquer alteração na estrutura corporal que não cause prejuízos para quaisquer órgãos ou sistemas humanos. $O$ músculo palmar longo (MPL), palmar menor ou pequeno palmar é um músculo caracterizado por possuir o ventre grosso e arredondado, e as extremidades afiladas, dando a ele a classificação de um músculo fusiforme. Este se localiza na região

\footnotetext{
${ }^{7}$ Fisioterapeuta Graduado pela Fundação de Educação e Cultura de Santa Fé do Sul - Santa Fé do Sul, Brasil. Especialista em Fisiologia do Exercício pela Faculdade Vale do Aporé - Cassilândia, Brasil. Mestre em Engenharia Biomédica pela Universidade Vale do Paraíba - São José dos Campos, Brasil. Professor do Curso de Medicina da Universidade Brasil - Fernandópolis, Brasil.
} 
anterior e superficial do antebraço e é considerado um músculo filogeneticamente em regressão (MOORE, 2012).

O MPL tem sua origem no epicôndilo medial do úmero e sua inserção no retináculo dos flexores, e na aponeurose palmar na extremidade proximal do segundo metacarpo. É o mais delgado dos músculos flexores do carpo e está recoberto pela fáscia do antebraço (MOORE, 2012).

Localiza-se medialmente ao músculo flexor radial do carpo, lateralmente ao músculo flexor ulnar e recobre posteriormente parte do músculo flexor superficial dos dedos. É irrigado por artérias ulnares recorrentes e inervado por um dos ramos do nervo mediano. Suas principais funções são tencionar a aponeurose palmar nos movimentos da mão e do polegar e auxiliar na flexão da articulação do punho juntamente com os músculos flexor radial do carpo, flexor ulnar do carpo, flexor dos dedos e do polegar e abdutor longo do polegar (MOORE, 2012).

Não há prejuízos na movimentação da mão em pessoas que não possuem esse músculo, porque o mesmo é um músculo secundário na flexão do carpo (GARCIA, 2005).

A figura 1 mostra vista ventral dos músculos superficiais do antebraço, demonstrando o músculo palmar longo e seu tendão. 
Figura 1 - Músculo Palmar Longo

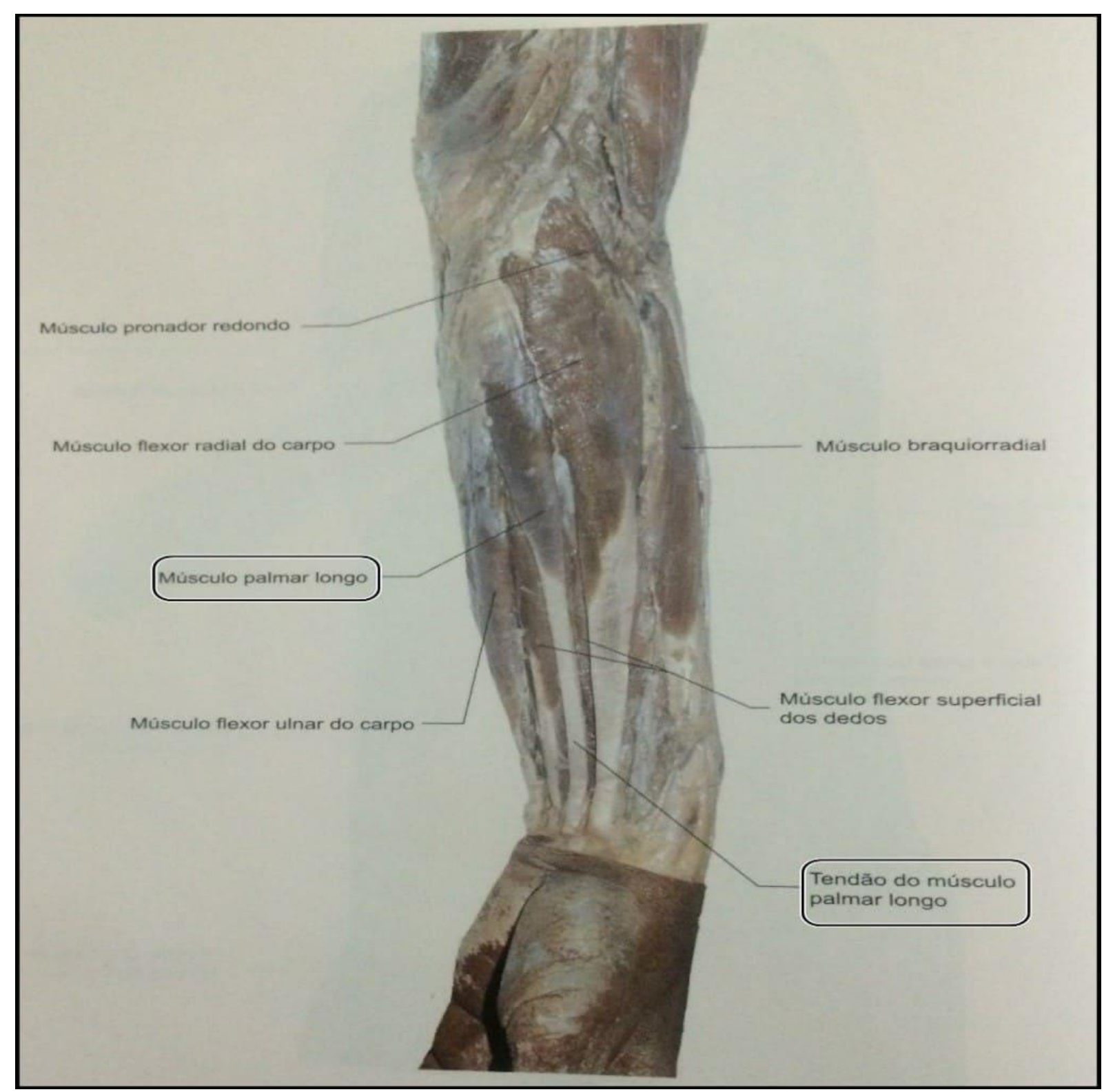

Fonte: Colicigno et al. (2.008, p. 124), adaptado pelas autoras.

Segundo Gardner (1971), a inexistência do MPL pode estar associada ao grupo racial, gênero sexual e a assimetria do corpo.

Atualmente existe grande interesse em conhecer a existência de seu tendão longo e único, pois ele tem sido utilizado em procedimentos cirúrgicos. Graças a esse músculo ocorrem cirurgias de auto enxerto muscular como plásticas reconstrutoras de mão, plásticas reconstrutoras de falhas labiais e mentuais, cirurgias ortopédicas de mão e 
tratamento para incontinência anal em crianças (PIERUCCI, 2013). Outras aplicações ocorrem em reconstruções ligamentares (KAUFMANN; PACEK, 2006), reconstrução de polias, defeitos oculares, reconstruções e ligamentos do dedo polegar e cotovelo, blefaropitoses (LAM, 1996).

Entendendo a importância desse músculo, esse estudo teve como objetivo analisar a variação anatômica do Músculo Palmar Longo em seres humanos encontrados em três estudos nacionais.

\section{METODOLOGIA}

Boente e Braga (2004) asseveram diferentes métodos de pesquisa, destaca-se, entre eles, a pesquisa acadêmica denominada como pesquisa cientifica, elaborada pelos discentes e traz como objetivo principal propiciar conhecimento e, como consequência, transformar este conhecimento em benefícios para a sociedade e para a academia.

Neste trabalho, inicialmente fez se a pesquisa bibliográfica, que é o início do processo investigativo do pesquisador, forma esta necessária para que se faça revisão bibliográfica sobre o tema em comento, além de aprofundar no conhecimento sobre o tema em tela a pesquisa bibliográfica assessora o pesquisador na escolha do método mais adequado quando se trata do conhecimento das variáveis analisadas e tendo conforto a qualidade e a autenticidade da pesquisa.

As fontes secundárias mais utilizadas pelos atores nesse processo trata-se de documentação confiável que pode ser acessada na sua forma apresentada dando oportunidade ao pesquisador de utilizá-lo para consulta, estudo ou prova, genericamente pode ser chamado, também, como referência.

Neste sentido a pesquisa documental que tem muita similaridade com a bibliográfica, tornando-a diferente quanto a natureza das fontes. Esta se vale de materiais que não receberam tratamento ou ainda podem ser reelaborados de acordo com os objetivos 
da pesquisa, portanto permite utilizar documentos que já foram analisados, mas que podem receber outras interpretações dos dados já levantados (GIL, 2010).

Dessa forma, nesse estudo foi utilizado como fontes secundárias: livros, periódicos, artigos, trabalhos monográficos e sites confiáveis, quanto aos documentos já existentes foram utilizados 3 artigos que tratam do assunto em tela submetidos a novas análises das autoras.

Foram analisados dados de três estudos realizados sobre a Prevalência do Músculo Palmar Longo em amostra populacional humana, todos utilizaram o método de Schaeffer para analisar a presença do músculo. O método consiste em realizar a oposição do primeiro e quinto dedo enquanto é realizada a flexão do punho e por meio desse movimento é possível observar o tendão do indivíduo sobre a pele, próximo a região anterior do punho, conforme Figura 2 que evidencia a presença do tendão do músculo. 
Figura 2 - Exposição do MPL pelo Método de Schaeffer.

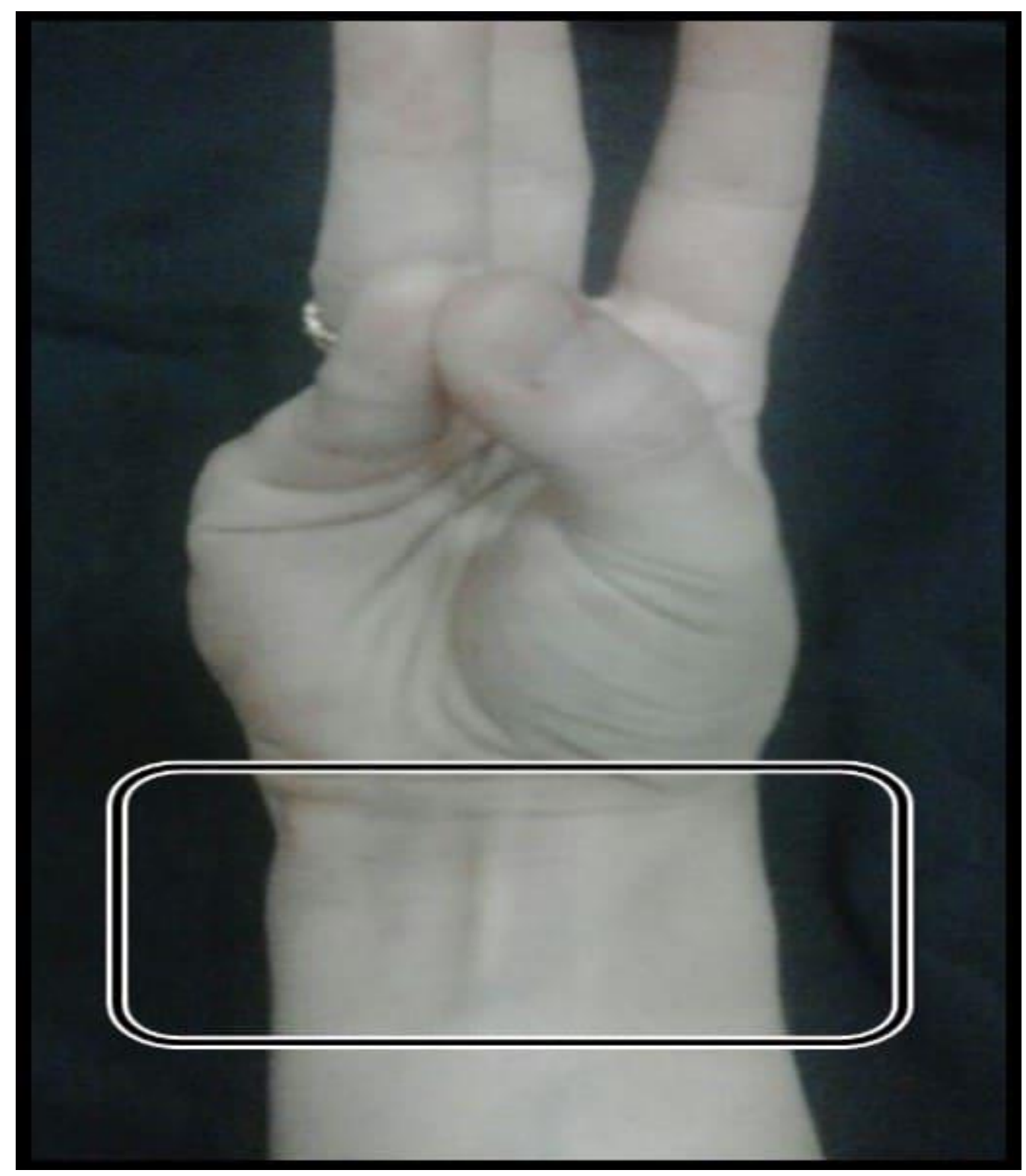

Fonte: Foto realizada pelas autoras.

No primeiro estudo, de autoria de Amauri Pierucci, Artur Ferreira Pereira e Maryane Oliveira Campos, foram analisados 240 discentes (480 antebraços) de graduação na Universidade Federal dos Vales Jequitinhonha e Mucuri (UFVJM), localizada em Diamantina no estado de Minas Gerais, dos quais 128 eram mulheres e 112 eram homens. 
No segundo estudo, de autoria de Lillian Bolonheis Garcia; Carolina Nechar Marques; Cinthya Mayumi dos Santos sob a orientação da Prof. Dra. Sônia Maria Marques Gomes Bertolini, foram analisados 200 voluntários (400 antebraços) de ambos os sexos de diferentes idades na cidade de Maringá - Paraná, dos amostrados 101 eram do sexo masculino e 99 do sexo feminino.

No terceiro estudo, de autoria de Maraiza silva Gomes; Janiffer soares Moraes; Milca Abda Morais; Carla Helrigle sob a orientação da Prof. Dra. Tais Malysz, realizado em Jataí - Goiás foram analisados 740 sujeitos (1.480 antebraços) de ambos os sexos, dos quais 461 eram mulheres e 279 eram homens.

\section{DISCUSSÃO E RESULTADOS}

No estudo 1 de Pierucci, Pereira e Campos (2013) os achados de pesquisa são apresentados no quadro 1 e gráfico 1 a seguir:

Quadro 1: Resultado extraído do Estudo 1.

\begin{tabular}{|c|c|c|c|}
\hline \multicolumn{4}{|c|}{ Estudo 1 } \\
\hline Situações & Mulheres & Homens & Total \\
\hline Presença do Músculo Palmar Longo & 100 & 90 & 190 \\
\hline Agenesia unilateral & 15 & 13 & 28 \\
\hline Agenesia bilateral & 13 & 9 & 22 \\
\hline Total & 128 & 112 & 240 \\
\hline
\end{tabular}

Fonte: Pierucci, Pereira e Campos (2013) - Adaptado pelas autoras.

Neste estudo constatou-se, conforme pode ser verificado no Quadro 1, que o MPL estava presente em 190 indivíduos, dos quais 90 eram do sexo masculino e 100 do feminino. A ausência unilateral foi presente em apenas 28 indivíduos, dos quais 13 
eram do sexo masculino e 15 do feminino. A agenesia bilateral foi observada em 22 indivíduos, dos quais 9 eram homens e 13 eram mulheres. Representando graficamente, tinha-se:

Gráfico 1 - Representação dos achados de pesquisa - Estudo 1.

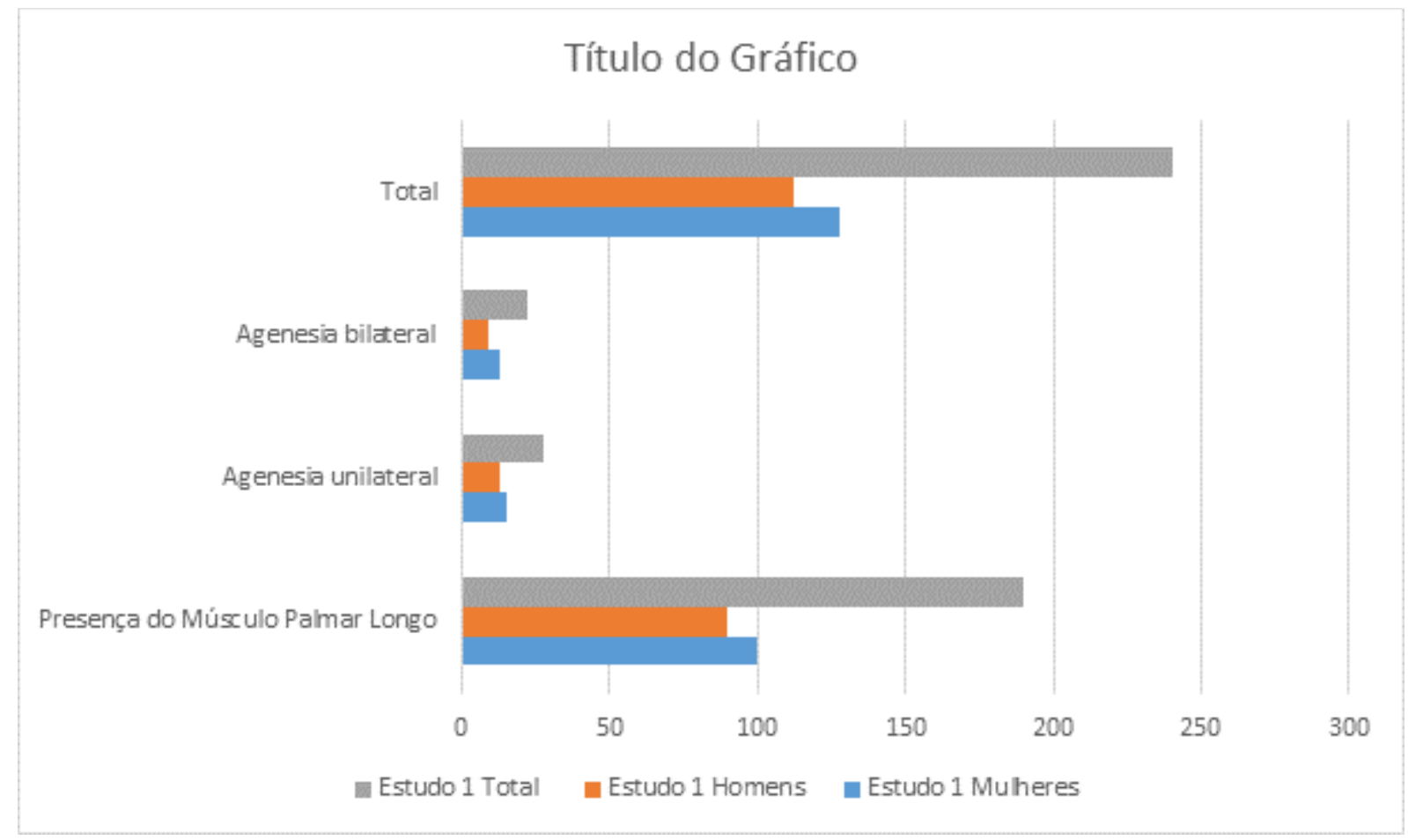

Fonte: Pierucci, Pereira e Campos (2013) - Adaptado pelas autoras.

Reordenando os achados de pesquisa obtém-se: 
Quadro 2: Resultado extraído do Estudo 1 - Participação ponderada de homens e mulheres

\begin{tabular}{|c|c|c|c|c|c|c|c|c|c|}
\hline \multicolumn{6}{|c|}{ Estudo 1} & \multicolumn{2}{|c|}{$\begin{array}{l}\text { Em relaçāo ao total de } \\
\text { mulheres e homes }\end{array}$} & \multicolumn{2}{|c|}{$\begin{array}{c}\text { Em relaçäo a } \\
\text { participação } \\
\text { pond erada de homens } \\
\text { e mulheres amostradas }\end{array}$} \\
\hline Situaçães & Mulheres & Homens & Total & $\begin{array}{l}\text { Mulheres } \\
\text { (\%) }\end{array}$ & $\begin{array}{l}\text { Homens } \\
(\%)\end{array}$ & $\begin{array}{l}\text { Mulheres } \\
\text { (\% tota) }\end{array}$ & $\begin{array}{l}\text { Homens } \\
(\% \text { total })\end{array}$ & $\begin{array}{l}\text { Mulheres } \\
(\% \text { total) }\end{array}$ & $\begin{array}{l}\text { Homens } \\
\text { (\% total) }\end{array}$ \\
\hline Presença do Músculo Palmar Longo & 100 & 90 & 190 & 78,1 & 80,4 & 41,7 & 37,5 & 36,5 & 42,9 \\
\hline Agenesia unilateral & 15 & 13 & 28 & 11,7 & 11,6 & 6,3 & 5,4 & 5,5 & 6,2 \\
\hline Agenesia bilateral & 13 & 9 & 22 & 10,2 & 8,0 & 5,4 & 3,8 & 4,7 & 4,3 \\
\hline Total & 128 & 112 & 240 & 100 & 100 & 10 & & 10 & $\%$ \\
\hline
\end{tabular}

Fonte: Pierucci, Pereira e Campos (2013) - Adaptado pelas autoras.

Verificou-se, que na amostra pesquisada, a presença do MPL tem maior incidência nos homens, representado por $80,4 \%$, constatado também, quando se considera a participação ponderada de ambos os sexos presentes na amostra analisada, representado por $42,9 \%$ dos pesquisados.

No estudo 2 de Garcia et al. (2005) os achados de pesquisa são apresentados no quadro 3 e gráfico 2 abaixo: 
Quadro 3 - Resultado extraído do Estudo 2.

\begin{tabular}{|c|c|c|c|}
\hline \multicolumn{4}{|c|}{ Estudo 2 } \\
\hline Situações & Mulheres & Homens & Total $^{*}$ \\
\hline Presença do Músculo Palmar Longo & 85 & 87 & 172 \\
\hline Agenesia unilateral & & & 24 \\
\hline Agenesia bilateral & & & 28 \\
\hline Total & 99 & 101 & 200 \\
\hline
\end{tabular}

Fonte: Garcia et al. (2005) - Adaptado pelas autoras

No estudo 2, o músculo foi constatado em 172 indivíduos, dos quais 87 eram homens e 85 eram mulheres, observou-se, na análise, agenesia unilateral em 24 casos, incluídos no total de indivíduos (172) considerados com a presença do MPL. Dos pesquisados, portanto, 28 apresentaram agenesia bilateral.

Representando-se graficamente, tinha-se: 
Gráfico 2 - Representação dos achados de pesquisa - Estudo 2.

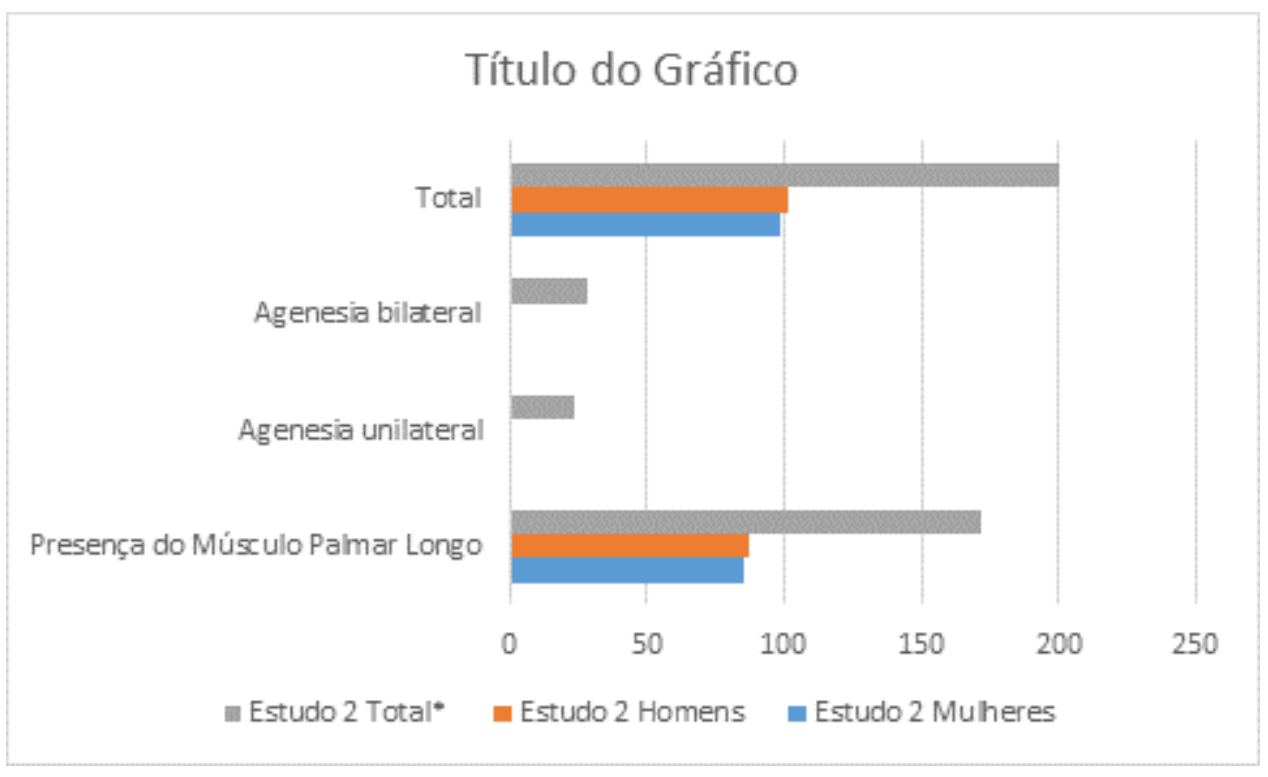

Fonte: Garcia et al. (2005) - Adaptado pelas autoras.

Reordenando dos achados de pesquisa obtém-se o quadro a seguir:

Quadro 4: Resultado extraído do Estudo 2.

\begin{tabular}{|c|c|c|c|c|c|}
\hline \multicolumn{7}{|c|}{ Estudo 2 } \\
\hline Situações & Mulheres & Homens & Total & $\begin{array}{c}\text { Mulheres } \\
(\%)\end{array}$ & $\begin{array}{c}\text { Homens } \\
(\%)\end{array}$ \\
\hline Presença do Músculo Palmar Longo & 85 & 87 & 172 & 85,9 & 86,1 \\
\hline Agenesia unilateral & \multicolumn{2}{|c|}{24} & 24 & \\
\hline Agenesia bilateral & \multicolumn{2}{|c|}{28} & 28 & \multicolumn{2}{|c|}{14} \\
\hline Total & 99 & 101 & 200 & 100 & 100 \\
\hline
\end{tabular}

Fonte: Garcia et al. (2005) - Adaptado pelas autoras.

Observando os achados de pesquisa verificou-se, que na amostra pesquisada, a presença do MPL tem maior incidência tanto nos homens como nas mulheres, enfatizando a presença do MPL na maioria dos indivíduos pesquisados. 
No estudo 3 de Gomes et al. (2011) os achados de pesquisa são apresentados no quadro 5 e gráfico 3 a seguir:

Quadro 5: Resultado extraído do Estudo 3.

\begin{tabular}{|c|c|c|c|}
\hline \multicolumn{4}{|c|}{ Estudo 3} \\
\hline Situações & Mulheres & Homens & Total \\
\hline Presença do Músc ulo Palmar Longo & 324 & 220 & 544 \\
\hline Agenesia unilateral & 77 & 29 & 106 \\
\hline Agenesia bilateral & 60 & 30 & 90 \\
\hline Total & 461 & 279 & 740 \\
\hline
\end{tabular}

Fonte: Gomes et al. (2011) - Adaptado pelas autoras.

No terceiro estudo o total amostrado era de 740 indivíduos, dos quais 461 eram mulheres e 279 eram homens, observou-se a presença bilateral em 544 indivíduos, 324 mulheres e 220 homens, enquanto apresentavam agenesia unilateral e bilateral, 106 (77 homens e 29 mulheres) e 90 (60 mulheres e 30 homens) indivíduos, respectivamente.

Representando-se graficamente, obteve-se: 
Gráfico 3 - Representação dos achados de pesquisa - Estudo 3.

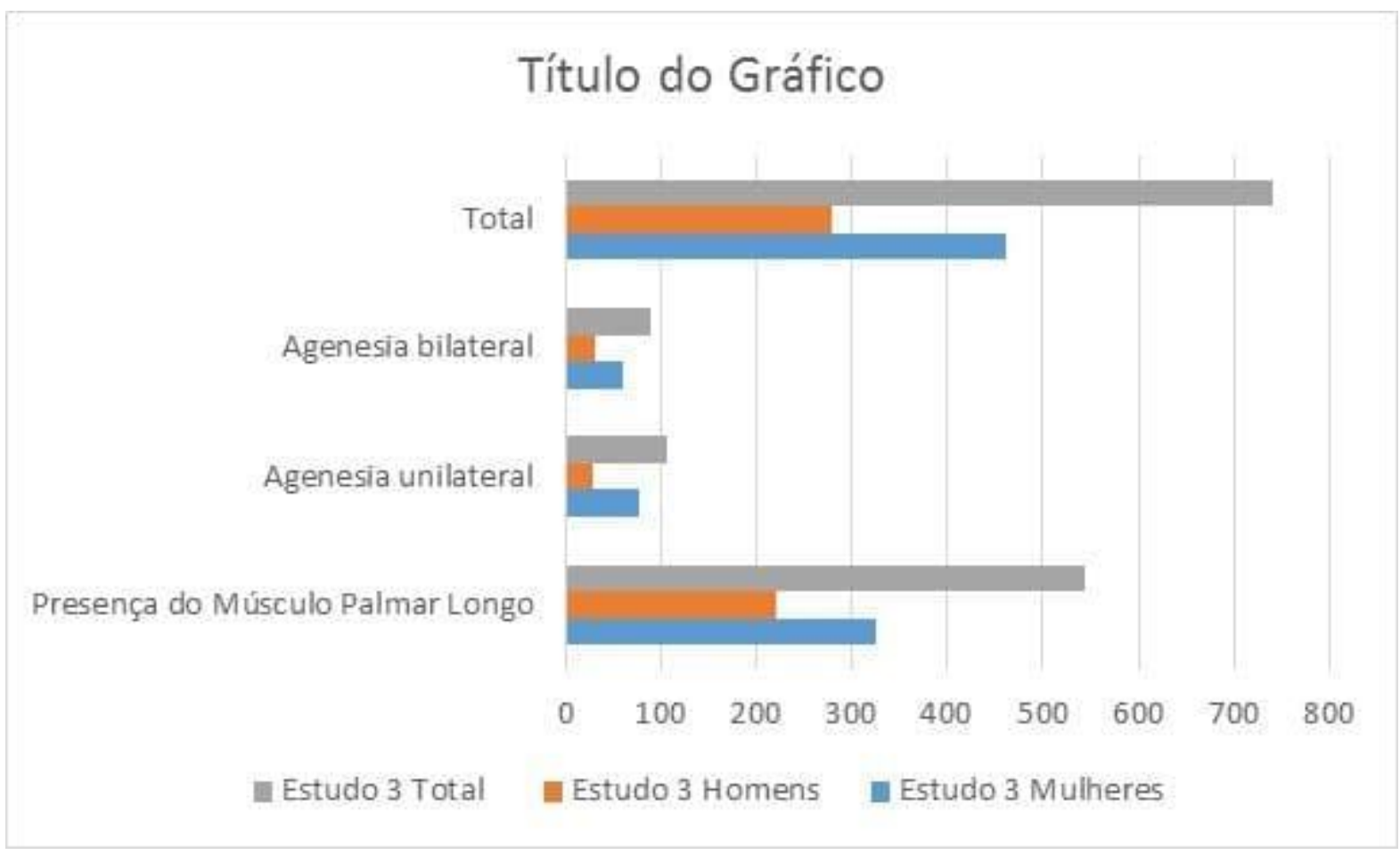

Fonte: Gomes et al. (2011) - Adaptado pelas autoras.

Reordenando os achados de pesquisa obtém-se o quadro a seguir: 
Quadro 6: Resultado extraído do Estudo 3.

\begin{tabular}{|c|c|c|c|c|c|c|c|c|c|}
\hline \multicolumn{6}{|c|}{ Estudo 3} & \multicolumn{2}{|c|}{$\begin{array}{l}\text { Em relaç̧̃o ao total de } \\
\text { mulherese homes }\end{array}$} & \multicolumn{2}{|c|}{$\begin{array}{c}\text { Em relaç̧̃o a } \\
\text { participaçăo } \\
\text { ponderada de homen } \\
\text { e mulheres amostrada: }\end{array}$} \\
\hline Situaçôes & Muheres & Homens & Total & $\begin{array}{l}\text { Muheres } \\
(\%)\end{array}$ & $\begin{array}{l}\text { Homens } \\
\text { (\%) }\end{array}$ & $\begin{array}{l}\text { Mulheres } \\
(\% \text { total }\end{array}$ & $\begin{array}{l}\text { Homens } \\
(\% \text { total) }\end{array}$ & \begin{tabular}{|l} 
Muhheres \\
(\% total)
\end{tabular} & $\begin{array}{l}\text { Homens } \\
(\% \text { total })\end{array}$ \\
\hline Presença do Múscubo Palmar Longo & 324 & 220 & 544 & 70,3 & 78,9 & 43,8 & 29,7 & 26,5 & 49,1 \\
\hline Agenesia unibteral & 77 & 29 & 106 & 16,7 & 10,4 & 10,4 & 3,9 & 6,3 & 6,5 \\
\hline Agenesia bilateral & 60 & 30 & 90 & 13,0 & 10,8 & 8,1 & 4,1 & 4,9 & 6,7 \\
\hline Total & 461 & 279 & 740 & 100,0 & 100,0 & 10 & $10 \%$ & & $0 \%$ \\
\hline
\end{tabular}

Fonte: Gomes et al. (2011) - Adaptado pelas autoras.

Observando os achados de pesquisa verifica-se, que na amostra pesquisada, a presença do MPL tem maior incidência nos homens, representado por $78,9 \%$, constatado também, quando se considera a participação ponderada de ambos os sexos presentes na amostra analisada, representado por $49,1 \%$ dos pesquisados.

Este artigo escrito a partir de 3 estudos encontrados na literatura técnica, retrabalhou os dados buscando conhecer a maior incidência do Músculo Palmar Longo em homens e mulheres, além de avaliar a existência deste nos indivíduos pesquisados.

Pelos testes realizados pode-se inferir que o MPL está presente na maioria dos indivíduos. Os três estudos demonstram que é comum as pessoas terem o músculo. O primeiro e o terceiro estudo evidenciaram que o sexo masculino é o que tem maior número de indivíduos com a existência do músculo.

A inexistência do músculo gera perda de força muscular funcionalmente insignificante, mas conforme preconiza Moore, Daley e Agur (2012, p. 744) "O músculo palmar longo está ausente em um ou ambos os lados em cerca de $14 \%$ das pessoas, mas suas 
ações são preservadas", essa afirmação do autor corrobora com os achados de pesquisa, principalmente nos estudos 1 e 3.

Corroborando ainda mais com o resultado da pesquisa dos estudos e adaptação realizada pelas autoras quando se tratou das ponderações entre o sexo dos amostrados Angelini et al. (2012) relata que por ser respeitado como um músculo auxiliar ou acessório e, portanto, não se caracteriza como essencial para funções do antebraço, visto que sua perda não acarreta perda de função, normalmente esse tendão é utilizado como enxerto em procedimentos cirúrgicos como lesões de tendões, recuperação de ligamentos, entre outros.

Mostrando a importância da existência do músculo nesse estudo, Scopel (2010, p.97) relata o uso do músculo para fixação de transplantes musculares na reanimação facial “(..) Durante o sorriso o tendão do músculo palmar longo limita o alongamento do músculo orbicular da boca paralisado e permite que as forças de tração do retalho muscular sejam transmitidas ao filtro e linha média do lábio inferior".

Conforme o apresentado pelos estudos analisados o mais comum é a existência do músculo nos indivíduos, tanto no sexo masculino como no feminino. Evidencia-se, ainda, que os estudos mostraram a existência com maior frequência do músculo no sexo masculino.

\section{CONCLUSÃO}

Conforme demonstrado nos indivíduos amostrados o MPL está presente na maioria dos indivíduos seja bilateralmente ou unilateralmente.

No primeiro estudo constatou-se que o músculo estava presente em $79,16 \%$ da população estudada. No segundo constatou-se sua presença em $86 \%$ do total da população em análise. No terceiro a presença bilateral foi observado em $73,5 \%$ do total. Embasados nos três estudos citados pode-se inferir que é comum possuir o músculo, quando comparados, o primeiro e o terceiro estudo citados, é possível inferir que no sexo masculino ocorre maior incidência do músculo. 
Observou-se, também, nos testes realizados e na literatura que a não existência do MPL é uma modificação corporal, não ocasionando dano aos seres humanos e, portanto, não devem ser consideradas como doenças, são, dessa forma, apenas variações anatômicas. Evidencia-se, ainda, pela literatura, sua importância em procedimentos cirúrgicos substituindo outros músculos essenciais aos seres humanos.

\section{REFERÊNCIAS}

ANGELINI JUNIOR, L. C.; ANGELINI, F.B.; OLIVEIRA, B.C.de; SOARES, S.A.; ANGELINI, L.C.;CABRAL, R.H. Utilização do tendão do músculo palmar longo em procedimentos cirúrgicos: estudo em cadáveres. Acta Ortop. Bras. vol.20 no.4 São Paulo 2012. Disponível em: http://dx.doi.org/10.1590/S1413-78522012000400007.

BOENTE, A.; BRAGA, G. P. Metodologia científica contemporânea para universitários e pesquisadores. Rio de Janeiro: Brasport, 2004.

BONSI, A. B. Estudo do musculo palmar longo numa população brasileira. 2000. [Tese de Doutorado]. Faculdade de Odontologia de Piracicaba, Universidade Estadual de Campinas. Piracicaba - SP.

COLIGNO, P.R.C.; Sacchetti, J.C.L.; Moraes, C.A.; Araújo, A. B. Atlas fotográfico de anatomia. Pearson Prentice Hall. São Paulo. 2009.

GARCIA, L.B; MARQUES, C.N; DOS SANTOS, C.M; BERTOLINI, S.M.M.G. Estudo da presença do músculo palmar longo e sua correlação com sexo e dominância motora. Iniciação Científica CESUMAR - Jan.jun. 2005, Vol. 07, n.01, pp. 19 - 24.

GARDNER, E.; GRAY, D.J.; O'RAHILLY, R. Anatomia Humana. 3eed., Rio de Janeiro, Guanabara Koogan, 1971, cap.4, p.27-35.

GIL, A.C. Como elaborar projetos de pesquisa. 5. ed. São Paulo: Atlas, 2010 
GOMES, M.S.; MORAES, J.S; MORAIS, M.A.; HELRIGLE, C.; MALYSZ, T. Estudo da Presença do Músculo Palmar Longo e sua Correlação com Sexo e Dominância Motora. 63 ${ }^{\text {a }}$ Reunião anual da SBPC - Sociedade Brasileira para o Progresso da Ciência 2011.

Disponível

em: http://www.sbpcnet.org.br/livro/63ra/resumos/resumos/2981.htm.

KAUFMANN, R.A.; PACEK, C.A. Pulley reconstruction using palmaris longus autograft after repeat trigger release. J Hand Surg Br. 2006 ;31(3):285-7.

LAM, D.S.; LAM, T.P.; CHEN, I.N.; TSANG, G.H.; GANDHI, S.R. Palmaris longus tendon as a new autogenous material for frontalis suspension surgery in adults. Eye (Lond). 1996;10 ( Pt 1):38-42.

MOORE, K.L.; DALLEY, A.F.; AGUR, A.M.R. Anatomia Orientada para a Clínica. 6. Ed. Rio de Janeiro: Guanabara Koogan, 2012.

MORAIS, M.A.; SANTOS, WG.; MALYSZ, T. Estudo da presença do músculo palmar longo e sua correlação com sexo e dominância motora. Jataí, GO, 2013.

PIERUCCI, A.; PEREIRA, A.F.; CAMPOS, M.O. Prevalência do músculo palmar longo em acadêmicos da Universidade Federal dos Vales do Jequitinhonha e Mucuri. Revista Unimontes Científica. Montes Claros, v. 15, n. 1 - jan. 2013. (ISSN 22365257).

SCOPEL, G.P. Estudo comparativo da fixação dos transplantes musculares na reanimação facial unilateral com e sem uso do tendão do palmar longo. [Tese de Doutorado]. Faculdade de Medicina da Universidade de São Paulo. 2010. Disponível em:http://www.modelarecirurgiaplastica.com.br/dr-gean-paulo-scopel.php, acesso em 12/10/2018.

SOBOTTA, J.W.; PAULSEN, F. Atlas de Anatomia Humana, volume 1. Ed.Guanabara, 23ª Edição. Rio de Janeiro. 2010.

Enviado: Abril, 2019. 
Aprovado: Julho, 2019. 